\title{
Testing the hypothesis of the influence of neutral turbulence on the deduction of ambipolar diffusivities from meteor trail expansion
}

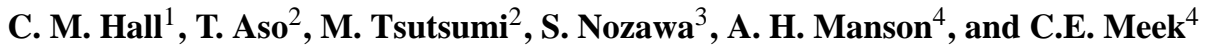 \\ ${ }^{1}$ Troms $\varnothing$ Geophysical Observatory, University of Troms $\varnothing$, N-9037 Troms $\varnothing$, Norway \\ ${ }^{2}$ National Institute of Polar Research, 1-9-10 Kaga, Itabashi, Tokyo 173-8515, Japan \\ ${ }^{3}$ STELab, Nagoya University Chikusa-ku, Nagoya 464-01, Japan \\ ${ }^{4}$ Inst. of Space and Atmospheric Studies, Univ. of Saskatchewan, Saskatoon, Saskatchewan S7N 5E2, Canada
}

Received: 21 October 2004 - Revised: 10 January 2005 - Accepted: 14 January 2005 - Published: 30 March 2005

\begin{abstract}
Fading times of radar echoes from underdense meteor trails in the upper mesosphere/lower thermosphere are commonly used to determine ambipolar diffusivities and hence ambient temperature. Diffusivities are generally expected to increase exponentially with height through the region from which the meteor trail echoes are obtained, viz., typically $70-110 \mathrm{~km}$ altitude for a $\sim 30-\mathrm{MHz}$ radar. In practice, however, this is more the exception: unexpectedly large diffusivities are obtained in the lower part of the regime, and unexpectedly low values are obtained in the upper part; only in the few kilometres on either side of the maximum in echo occurrence (viz., $90 \mathrm{~km}$ for a $30-\mathrm{MHz}$ radar) does the diffusivity profile behave as expected. Hall (2002) hypothesised that neutral turbulence might be enhancing expansion of the meteor trail in the lower part of the regime. In this communication, due to results only available since the publication of Hall's suggestion, we are able to refute the hypothesis.
\end{abstract}

Keywords. Atmospheric composition and structure (Instrumentation and techniques) - Meteorology and atmospheric dynamics (Turbulence; Middle atmosphere dynamics)

\section{Introduction}

The Nippon/Norway Svalbard Meteor Radar (NSMR) came into operation in spring 2001 and has since been described by Hall et al. (2002). A thorough explanation of the operation of such a radar has recently been given by Holdsworth et al. (2004), including descriptions of the physical atmospheric parameters that may be derived and how this is done, along with further references to the underlying theory. Observing the decay of echoes from meteor trails enables us to esti-

Correspondence to: C. M. Hall

(chris.hall@tgo.uit.no) mate the ambipolar diffusion coefficient of ions in the trail. It might be anticipated that this diffusivity would exhibit an exponential increase with height, however Hall (2002) noticed that this was often not the case, as had also been observed by, for example, Dyrud et al. (2001) in results from other meteor radar. While the latter paper concentrated on the less-than-expected diffusivities in the upper part of the echo height regime (i.e. some $\mathrm{km}$ above the altitude of peak echo occurrence of, for example, $\sim 90 \mathrm{~km}$ for a $\sim 30-\mathrm{MHz}$ radar) and the mechanism for this behaviour, Hall (2002) examined the more-than-expected values in the lower part of the regime. By examining time scales of diffusion processes in the atmosphere, Hall (2002) found a possibility that the fading of radar echoes might be augmented by neutral air turbulence, giving rise to an overestimation of diffusivity. In other terms, it seemed possible that the diffusivity estimated from the radar might be a combination of both molecular and turbulent processes. In fact, Brown (1976) suggested that use of radar frequencies lower than $30 \mathrm{MHz}$ might be employed to investigate the very effect of turbulence on meteor train echoes.

The absence of any independent determination of neutral air turbulence in the same atmospheric volume as monitored by NSMR precluded a straightforward ratification by Hall of his (2002) hypothesis. However, since the beginning of 2004 the Troms $\varnothing$ MF radar (Hall, 2001) and the newly established Nippon/Norway Troms $\varnothing$ Meteor Radar (NTMR) have been observing simultaneously. NTMR is, for all intents and purposes, identical to NSMR and therefore similarly described by Holdsworth et al. (2004). Hall and Husøy (2004) compared altitudes of tidal phases observed by the MFR and NTMR respectively, confirming the conclusion using careful measurements of delays in the MFR system (employing a delay line to resolve ambiguities), and therefore resolving the earlier uncertainty in the MFR altitude calibration. Thus, 


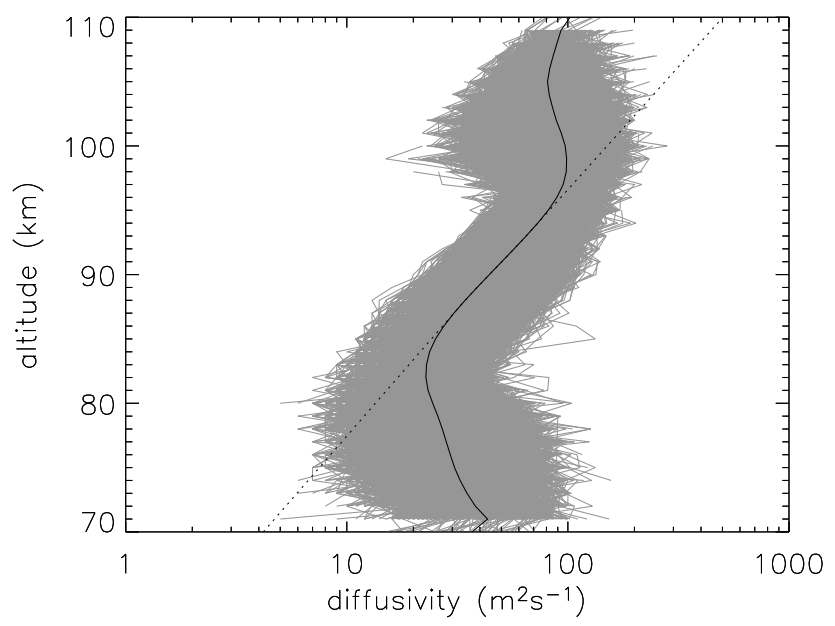

Fig. 1. Diffusion coefficients delivered by NTMR. Every available 30-minute average profile from 1 January to 30 June 2004 has been superimposed (grey lines) and then averaged to obtain a 6-month average (solid black line). The peak meteor trail echo occurrence height is at $\sim 90 \mathrm{~km}$. Departures from the anticipated exponential increase with height of molecular diffusion (e.g. dotted black line) are discussed by Dyrud et al. (2001) above $\sim 95 \mathrm{~km}$, and by Hall (2002) below $\sim 85 \mathrm{~km}$.

a correlation between the departure of the diffusion coefficient yielded by NTMR, from the expected exponential profile, with estimates of the turbulent energy dissipation rate obtained from the MFR (Hall et al., 1998), would be a prima facie ratification the Hall (2002) mechanism.

\section{Results}

Let us first review the signature of the diffusion coefficient profiles that aroused the concern of at least Hall (2002), Dyrud et al. (2001) and for that matter, Hocking (private communication, 2002). Here, in Fig. 1 we show results from NTMR. All available 30-min average profiles for the first 6 months of 2004 are overlaid (grey), and we see clearly the tendencies toward decreasing values with increasing height above $95 \mathrm{~km}$ and increasing values with decreasing height below $82 \mathrm{~km}$. The average (i.e. 6-month average) of these is also shown (solid black). We see that the average profile at least exhibits an exponential increase with altitude in the range $85-95 \mathrm{~km}$, and a linear fit has been performed in log space in order to illustrate how the average diffusion coefficient profile might be expected to look (dotted black). Obviously, this could have been done for each individual 30-min profile, but the resulting figure would have been intractable. To elucidate our nomenclature, we have indeed used the term "diffusion coefficient" to describe the parameter illustrated in Fig. 1, but omit to qualify it with any specific diffusion mechanism.

To determine the departures of the profiles from what we assume to be the molecular diffusivity we would need to fit exponential profiles (or in practice linear fits in log space, as
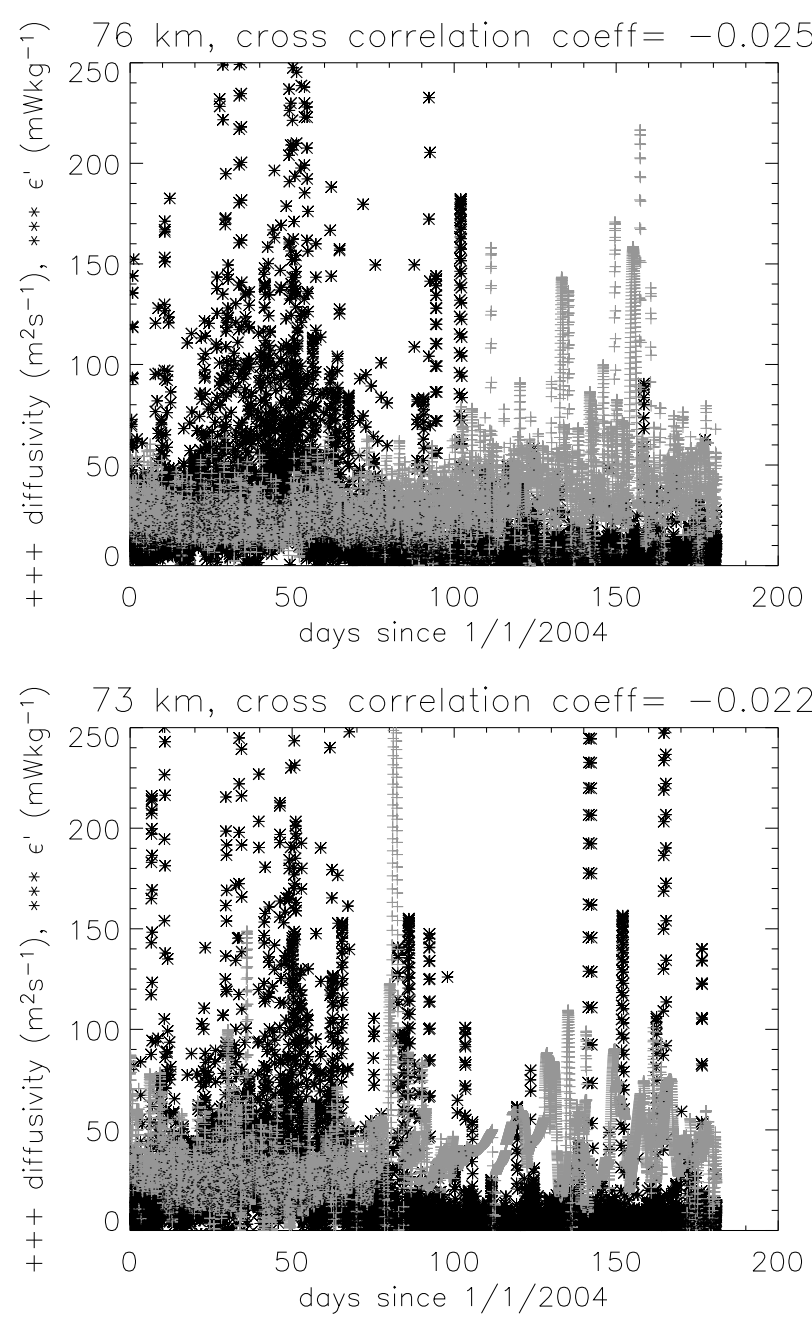

Fig. 2. Time series of estimates of neutral air turbulent energy dissipation rate $\left(\varepsilon^{\prime}\right)$ obtained by the Troms $\emptyset$ MF radar (black asterisks) and the diffusion coefficient values found from meteor trail echo fading times (grey pluses). Upper panel: $76 \mathrm{~km}$; lower panel: $73 \mathrm{~km}$.

in the figure) to selected height intervals of the 30-min profiles and extrapolate these to the lower, offending altitudes. Experience has shown, however, that this is not always successful due to noisy and occasionally sparse data, particularly when automated. If the hypothesis of Hall (2002) is valid, it is indeed the departures of the diffusivity profiles from those of molecular diffusivity that would be correlated with turbulent intensity. However, examination of the average profiles in the figure reveals that at $70 \mathrm{~km}$ the molecular diffusion represents only $10 \%$ of the total (if the average profile is indeed one of diffusion) and at $75 \mathrm{~km}$ the molecular contribution is still only $25 \%$. Thus, if the turbulent intensity is compared to the absolute supposed diffusion coefficient rather than the departure from a somewhat uncertain determination of the molecular diffusion coefficient, a correlation, decreasing with altitude, should still be evident if the Hall (2002) mechanism is valid. 
In order to simplify comparison between the MFR and NTMR results, we have interpolated both the turbulent energy dissipation rate estimate, $\varepsilon$ ' from the former and the diffusion coefficient from the latter, to a regular 30-min grid from 1 January to 30 June 2004. We have then superimposed the resulting time series at the selected altitudes of 73 and $76 \mathrm{~km}$ (data at $70 \mathrm{~km}$ are somewhat sparse, and above $76 \mathrm{~km}$ the measured diffusivity and the presumed molecular diffusivities begin to converge), and plotted the results in Fig. 2. Although not a study here, the turbulent energy dissipation estimate (black asterisks) exhibits lower values during the summer as expected according to, for example, Lübken (1997). On the other hand, it is difficult to identify any clear seasonal trend in the diffusivity (grey pluses), although the known temperature variation in the mesosphere (e.g. Lübken and von Zahn, 1991) should dictate this. We assert that, from a visual comparison of these time series, there is no correlation whatsoever, either at $76 \mathrm{~km}$, or at, following Hall (2002) theoretically the more likely, $73 \mathrm{~km}$. Furthermore, cross correlating these time series at zero lag, we obtain correlation coefficients of -0.022 and -0.025 at 73 and $76 \mathrm{~km}$ respectively. Since the time resolution of the MFR data is $5 \mathrm{~min}$ and that of NTMR is $30 \mathrm{~min}$ and the time scales for echo fading times are of the order of seconds only, only a zero lag cross correlation is meaningful.

\section{Conclusion}

Hall (2002) proposed that neutral turbulence might be responsible for reducing meteor trail echo delay times and thus leading to observed overestimates of molecular diffusivity. Other mechanisms for such overestimates include incorrect determination of echo altitude due to combinations of incorrect angle-of-arrival and range determination, and incorrect determination of fading time, conceivably due to inappropriate sampling of the individual echoes. Here, we conclude that there exists absolutely no correlation between neutral air turbulent intensity, parameterized by estimates of energy dissipation rate, and the values of diffusion coefficient delivered by a $30-\mathrm{MHz}$ meteor radar, where the latter values show a significant departure from the molecular diffusivities indicated by a downward extrapolation of the information from around the peak echo occurrence height. We have thus tested the suggestion of Hall (2002) that under certain conditions neutral air turbulence may give rise to an overestimation of molecular diffusivity by meteor radar operating at $30 \mathrm{MHz}$, and reject the hypothesis. At the same time, however, we cannot eliminate the possibility of turbulence affecting decay times for other frequencies.
Acknowledgements. The first author thanks the Norwegian Research Council for support. The NIPR authors thank the Ministry of Education, Science and Technology for the Grant-in-aid money on the Arctic upper atmosphere coupling study in constructing the NTMR meteor radar system. The Canadian authors gratefully acknowledge grants from the University of Saskatchewan, and the Institute of Space and Atmospheric Studies and the national agency, NSERC.

Topical Editor U.-P. Hoppe thanks a referee for his/her help in evaluating this paper.

\section{References}

Brown, N.: Radio echoes from meteor trains at a radio frequency of $1.98 \mathrm{MHz}$, J. Atmos. Terr. Phys., 38, 83-87, 1976.

Dyrud, L. P., Oppenheim, M. M., and vom Endt, A. F.: The anomalous diffusion of meteor trails, Geophys. Res. Lett., 28, 27752778, 2001.

Hall, C. M., Meek, C. E., and Manson, A. H.: Turbulent energy dissipation rates from the University of Tromsø/University of Saskachewan MF radar,J. Atmos Solar Terr. Phys, 60, 437-440, 1998.

Hall, C. M.: The Ramfjormoen MF radar $\left(69^{\circ} \mathrm{N}, 19^{\circ} \mathrm{E}\right)$ : Application development 1990-2000, J. Atmos. Solar-Terr. Phys., 63, 171-179, 2001.

Hall, C. M.: On the influence of neutral turbulence ambipolar diffusivities deduced from meteor trail expansion, Ann. Geophys., 20, 1857-1862, 2002.

Hall, C. M., Aso, T., and Tsutsumi, M.: An examination of high latitude upper mesosphere dynamic stability using the Nippon /Norway Svalbard Meteor Radar, Geophys. Res. Lett., 29, 12111213, 2002.

Hall, C. M. and Husøy, B. O.: Altitude calibration of the Troms $\varnothing$ Medium Frequency Radar, Troms $\emptyset$ Geophysical Observatory Report, ISSN:1504-1174, 13, 2004.

Holdsworth, D. A., Reid, I. M., and Cervera, M. A.: Buckland Park all-sky interferometric meteor radar, Radio Sci., 39, RS5009, doi:10.1029/2003RS003014, 2004.

Lübken, F.-J.: Seasonal variation of turbulent energy dissipation rates at high latitudes as determined by in situ measurements of neutral density fluctuations, J. Geophys. Res., 102, 1344113 456, 1997.

Lübken, F.-J. and von Zahn, U.: Thermal structure of the mesopause region at polar latitudes, J. Geophys. Res., 96, 20 841-20 857, 1991. 